\title{
A network pharmacology approach to discover active compounds and action mechanisms of San-Cao Granule for treatment of liver fibrosis
}

This article was published in the following Dove Press journal:

Drug Design, Development and Therapy

19 February 2016

Number of times this article has been viewed

Shizhang Wei, ${ }^{1,2}$ Ming Niu, ${ }^{3}$ Jian Wang, ${ }^{2}$ Jiabo Wang, ${ }^{3}$ Haibin $\mathrm{Su},{ }^{4}$ Shengqiang Luo, ${ }^{5}$ Xiaomei Zhang, ${ }^{2,6}$ Yanlei Guo, ${ }^{6}$ Liping Liu,' Fengqun Liu,' Qingguo Zhao,' Hongge Chen,' Xiaohe Xiao, ${ }^{3}$ Pan Zhao, ${ }^{4,7}$ Yanling Zhao $^{1,2}$

'Department of Pharmacy, 302 Hospital of People's Liberation Army, Beijing, People's Republic of China; ${ }^{2}$ hharmacy College, Chengdu University of Traditional Chinese Medicine, Chengdu, People's Republic of China; ${ }^{3}$ China Military Institute of Chinese Medicine, 302 Hospital of People's Liberation Army, Beijing, People's Republic of China; ${ }^{4}$ Liver Failure Therapy and Research Center, 302 Hospital of People's Liberation Army, Beijing, People's Republic of China; ${ }^{5}$ Department of Integrative Medical Center, 302 Hospital of People's Liberation Army, Beijing, People's Republic of China; ${ }^{6}$ Chong Qing Academy of Chinese Traditional Materia Medica, Key Laboratory of Chongqing TCM Resources, Chongqing, People's Republic of China; ${ }^{7}$ Clinical Trial Center, 302 Hospital of People's Liberation Army, Beijing, People's Republic of China

Correspondence: Yanling Zhao

Department of Pharmacy, 302 Hospital of People's Liberation Army, 100 Western 4th Ring Road, Beijing I00039,

People's Republic of China

Email zhaoyl2855@|26.com

Pan Zhao

Clinical Trial Center, 302 Hospital of People's Liberation Army, 100 Western 4th Ring Road, Beijing 100039,

People's Republic of China

Email doczhaopan@I26.com
Ethnopharmacological relevance: San-Cao Granule (SCG) has been used in patients with liver fibrosis for many years and has shown good effect. However, its mechanism of therapeutic action is not clear because of its complex chemical system. The purpose of our study is to establish a comprehensive and systemic method that can predict the mechanism of action of SCG in antihepatic fibrosis.

Materials and methods: In this study, a "compound-target-disease" network was constructed by combining the SCG-specific and liver fibrosis-specific target proteins with protein-protein interactions, and network pharmacology was used to screen out the underlying targets and mechanisms of SCG for treatment of liver fibrosis. Then, some key molecules of the enriched pathway were chosen to verify the effects of SCG on liver fibrosis induced by thioacetamide (TAA).

Results: This systematic approach had successfully revealed that 16 targets related to 11 SCG compounds were closely associated with liver fibrosis therapy. The pathway-enrichment analysis of them showed that the TGF- $\beta 1 /$ Smad signaling pathway is relatively important. Animal experiments also proved that SCG could significantly ameliorate liver fibrosis by inhibiting the TGF- $\beta 1 /$ Smad pathway.

Conclusion: SCG could alleviate liver fibrosis through the molecular mechanisms predicted by network pharmacology. Furthermore, network pharmacology could provide deep insight into the pharmacological mechanisms of Chinese herbal formulas.

Keywords: San-Cao Granule, network pharmacology, liver fibrosis, TAA

\section{Introduction}

Liver fibrosis, a common pathological change of chronic liver injury induced by various etiological factors, is the early stage of cirrhosis. ${ }^{1,2}$ An important feature of liver fibrosis is the massive deposition of extracellular matrix (ECM) proteins. Activated hepatic stellate cells (HSCs) are the major source of ECM, which regulate the massive production and secretion of ECM. ${ }^{3}$ Although liver fibrosis is a common and serious disease, there has been no satisfactory treatment.

In recent years, the therapy against hepatic fibrosis with traditional Chinese medicines (TCMs) in the People's Republic of China has achieved great progress. ${ }^{4}$ Chinese herbal formulas including more than two kinds of TCM have synergistic therapeutic efficacy because of multiple active compounds; hence, an increasing number of Chinese herbal formulas have been researched for their effect on antihepatic fibrosis. ${ }^{5-7}$

SCG consists of Rubia cordifolia L., Siegesbeckia orientalis L., Gentiana macrophylla Pall., Salvia miltiorrhiza Bunge, Forsythia suspensa (Thunb.) Vahl., and Glycyrrhiza uralensis Fisch. in the ratio of $3: 3: 1.5: 1.5: 1.5: 1$ and has been used to 


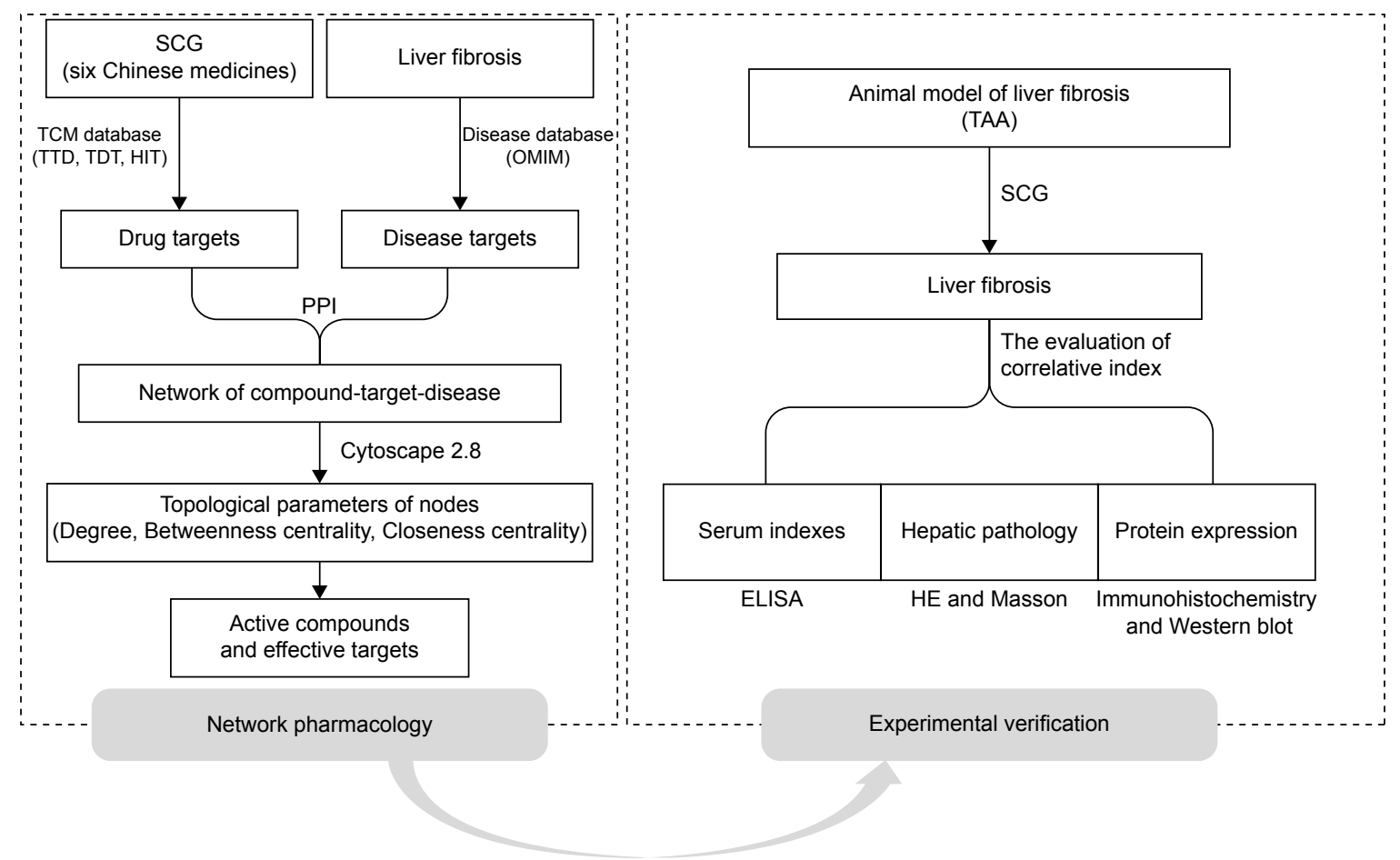

Figure I The whole framework of this study based on an integrative strategy of network pharmacology and experimental verification.

Abbreviations: SCG, San-Cao Granule; TAA, thioacetamide; ELISA, enzyme-linked immunosorbent assay; HE, hematoxylin-eosin; TTD, Therapeutic Target Database; TDT, TCM Database@Taiwan; HIT, Herbal Ingredients' Targets Database; OMIM, Online Mendelian Inheritance in Man; PPI, protein-protein interaction.

treat liver fibrosis. Its clinical efficacy has been confirmed in hepatic fibrosis patients, but the mechanisms of action, precise targets, and the relationships between these herbs and diseases are still unanswered. Therefore, further research of SCG remains a big challenge for us.

Recently, network pharmacology has attracted the attention of Chinese medicine researchers because it can be used to analyze the relationship between drug and disease using network from a proteome or at systematic level. ${ }^{8}$ Especially, it can react and clarify the interactive relationship between multiple components and multiple targets of TCM and has become a research focus of the theory and method in drug discovery and development. ${ }^{9}$ Therefore, we applied network pharmacology in our study to understand the efficacy of SCG in the prevention of liver fibrosis. Then, we validated the results obtained from the forecast of network pharmacology and finally developed a new method for the exploration of the action mechanisms of Chinese herbal formula to promote its modernization and globalization (Figure 1).

\section{Materials and methods Database construction}

The chemical structures of the composite compounds in SCG were obtained from TCM Database@Taiwan (TDT). ${ }^{10}$ Known compound targets were collected from Herbal
Ingredients' Targets Database (HIT), ${ }^{11}$ and the putative targets from these were screened out from Therapeutic Targets Database (TTD) $)^{12}$ through structural similarity comparison. Gene and protein targets associated with liver fibrosis therapy were collected from the Online Mendelian Inheritance in Man (OMIM) ${ }^{13}$ database. ${ }^{14}$ Other interacting human proteins of the aforementioned targets were obtained from Database of Interacting Proteins (DIP), and different ID types of the proteins were converted to UniProt IDs.

\section{Network construction and analysis}

To provide the scientific and reasonable interpretation of the complex relationships between the compounds and targets associated with liver fibrosis, network analysis was performed. The chemical components, SCG putative targets, liver fibrosis-significant targets, and interacting proteins were all connected to construct a "compound-target-disease" network by using protein-protein interaction (PPI) information. Cytoscape 2.8.3 was applied to visualize and analyze the network, and the topological features of each node in the network were calculated using CentiScaPe 1.2 of the Cytoscape software. Only the nodes with higher values of "Degree", "Betweenness centrality", and "Closeness centrality" (above twofold of the median value) were identified as the candidate SCG targets for liver fibrosis. ${ }^{15}$ 


\section{Drugs and reagents}

SCG consisted of Rubia cordifolia L., Siegesbeckia orientalis L., Gentiana macrophylla Pall., Salvia miltiorrhiza Bunge, Forsythia suspensa (Thunb.) Vahl and Glycyrrhiza uralensis Fisch., which were produced by Beijing Sheng Shi Long Herbs Co., Ltd (Beijing, People's Republic of China). The aforementioned TCMs were extracted by the following process: crude medical material was extracted with ten-fold the volume of water, twice, 1 hour for each time. The extract was concentrated and was then purified with alcohol. The supernatant through purification was condensed and dried, then the dry powder was mixed with moderate dextrin and pelletized with $95 \%$ alcohol.

Thioacetamide (TAA) was purchased from Sinopharm Chemical Reagent Co., Ltd (Shanghai, People's Republic of China). Serum alanine transaminase (ALT), aspartate aminotransferase (AST), and albumin (ALB) testing kits were purchased from Nanjing Jiancheng Co. Ltd (Nanjing, People's Republic of China). Serum total bilirubin (TBIL), hydroxyproline (Hyp), hyaluronic acid (HA), laminim (LN), and type IV collagen (CIV) testing kits were purchased from Clong-Cloud Corp (Houston, TX, USA); $\alpha$-SMA, CTGF, TGF- $\beta 1$, p-Smad3, Smad3, and Smad7 primary antibodies were purchased from Abcam (Cambridge, UK).

\section{Time-of-flight liquid chromatography mass spectrometry mass spectrometer system}

The conditions of LC (SHIMADZU,LC-30AD) were as follows: column - Kinetex 00D-4462-AN C18 $(100 \times 2.1,2.6 \mu \mathrm{m})$; mobile phase - acetonitrile with $0.1 \%(\mathrm{v} / \mathrm{v})$ formic acid (A) and water containing $0.1 \%(\mathrm{v} / \mathrm{v})$ formic acid $(\mathrm{B})$; gradient 0-1 minutes, 5:95; 1-8 minutes, 80:20; 8-15 minutes, 95:5; 15-20 minutes, $100: 0(\mathrm{~A}: \mathrm{B}, \mathrm{v} / \mathrm{v})$; column temperature $-35^{\circ} \mathrm{C}$; flow rate $-0.3 \mathrm{~mL} / \mathrm{min}$; and injection volume $-3 \mu \mathrm{L}$.

The TOF-MS (Triple TOF 4600) parameters were as follows: Curtain Gas: 25.000; Ion Source Gas 1:55.000; Ion Source Gas2:55.000; Ionspray Voltage Floating: 4,500.000; Temperature: 600.000 .

\section{Animals}

Male Sprague Dawley rats $(180 \pm 10 \mathrm{~g})$ were obtained from the animal center of Military Medical Sciences Academy of the PLA (Permission no SCXK-(A) 2012-0004). The rats were acclimatized for 1 week under environmentally controlled conditions with temperature $\left(25^{\circ} \mathrm{C} \pm 0.5^{\circ} \mathrm{C}\right)$, humidity $(55 \% \pm 5 \%)$, and light (12:12 hour light:dark cycle) prior to the experiment. The animals received humane care in compliance with the Chinese Animal Protection Act, according to the National Research Council criteria. The study was approved by the ethics committee of Beijing 302 Hospital.

\section{TAA-induced liver fibrosis and treatments}

TAA can induce liver fibrosis in Sprague Dawley rats. Ursodeoxycholic acid (UDCA) was taken as a positive medicine, because it is an accepted drug for treating liver fibrosis in clinical application and laboratory studies. In our study, 60 Sprague Dawley rats were divided into normal group (saline-treated), TAA-treated, TAA-treated + UDCA $(60 \mathrm{mg} / \mathrm{kg})$, TAA-treated + SCG $(3.6 \mathrm{~g} / \mathrm{kg})$, TAA-treated + SCG $(1.8 \mathrm{~g} / \mathrm{kg})$, and TAAtreated $+\mathrm{SCG}(0.8 \mathrm{~g} / \mathrm{kg})$ groups, with ten rats in each group. TAA was dissolved in saline and then intraperitoneally administered $(200 \mathrm{mg} / \mathrm{kg})$ to all the rats except the normal group twice a week for 6 weeks, and then the rats were orally administered with the corresponding drugs daily for 4 weeks. During the drug administration, TAA was continually administered once a week until the end of the experiment. All the animals were fasted for 24 hours before they were sacrificed.

\section{Evaluation of serum and tissue indexes}

The effect of SCG on liver injury and liver fibrosis induced by TAA was evaluated using serum ALT, AST, ALB, HA, LN, CIV, and tissue Hyp testing kits through microplate test or enzyme-linked immunosorbent assay.

\section{Histopathological and immunohistochemical evaluation}

The left lobe of livers of all rats were excised and fixed in $4 \%$ paraformaldehyde buffer for 24 hours. Then, the fixed liver tissues were dehydrated, embedded in paraffin, and sectioned into $4 \mu \mathrm{m}$ thick sections. The section was stained with hematoxylin and eosin (HE) and Masson fiber stains. These experimental operations were performed by a professional pathologist (LuYinglin, Department of Pathology, Academy of Military Medical Sciences, Beijing, People's Republic of China). Hepatic fibrosis was quantitatively assessed by analyzing Masson-stained paraffin sections under an optical microscope. The degree of liver fibrosis was graded based on the following criteria: 0: no fibrosis; I: slight fibrosis, fibrosis located in the central liver lobule; II: moderate fibrosis, widened central fibrosis; III: severe fibrosis, fibrosis extended to the edge of liver lobule; and IV: liver cirrhosis. ${ }^{16}$ The percentage of collagen content (collagen area/total area) was measured using the Image software (Image pro-plus 6.0, Media Cybernetics, Inc., Rockville, MD, USA). 
For the immunohistochemical analysis of $\alpha$-SMA, the $5 \mu \mathrm{m}$ liver sections were dipped in 3\% hydrogen peroxide for 30 minutes to quench endogenous peroxidase activity after being dewaxed and hydrated, and then were blocked and incubated overnight at $4^{\circ} \mathrm{C}$ with anti- $\alpha$-SMA antibody. A detectable color was generated after being incubated with 3, 30-diaminobenzidine tetrachloride for 5 to 10 minutes. The morphological characteristics were visualized by light microscopy. The integrated optical density (IOD) value of $\alpha$-SMA was detected using the Image software (Image proplus 6.0).

\section{Western blotting}

To detect the levels of protein expression of $\alpha$-SMA, TGF- $\beta 1$, p-Smad3, Smad3, Smad7, and CTGF, we performed Western blotting. The liver tissues $(50 \mathrm{mg})$ stored in liquid nitrogen tank were homogenized in $450 \mathrm{~mL}$ PBS with proteinase inhibitor, and were then centrifuged at $12,000 \times g$ at $4^{\circ} \mathrm{C}$ for 10 minutes to obtain a supernatant. The total protein obtained was quantified using the BCA protein assay kit (Thermo Fisher Scientific, Waltham, MA, USA). The protein sample was boiled for 10 minutes. Next, equal amounts of protein sample were run on $12 \%$ SDS-PAGE, transferred onto polyvinylidene fluoride (PVDF) membrane, and incubated in blocking buffer (TBST; $5 \times$ TBS: Tween-20 =1:20 (V/V)) nonfat milk for 1-2 hours. The PVDF membrane was then incubated with anti- $\alpha-\operatorname{SMA}(1: 2,000)$, CTGF (1:500), anti-TGF- $\beta 1$ (1:50), anti-p-Smad3 (1:500), anti-Smad3 $(1: 3,000)$, and anti-Smad7 $(1: 2,000)$ primary antibodies for 3 hours, respectively, and then washed three times with TBST. The PVDF membrane was incubated with secondary HRP-conjugated donkey anti-mouse IgG antibody $(1: 2,000)$ for 45 minutes and was washed three times again. The bound antibody was reacted with the chemiluminescence kit, and the intensity of the protein bands was analyzed with the ImageJ software program.

\section{Statistical analysis}

The data of the experiment are presented as means \pm SEM. One-way ANOVA was used for statistical analysis. $P<0.01$ was regarded as highly significant and $P<0.05$ was regarded as significantly different.

\section{Results}

\section{Targets screening of SCG and liver fibrosis}

Based on database construction method, a total of 68 compounds and 387 targets of SCG, 59 candidate protein targets associated with liver fibrosis therapy, and 440 interacting proteins of the targets were screened out. Among the compounds and targets of SCG, 16 compounds and 51 targets were collected for Rubia cordifolia L., 24 compounds and 75 targets for Siegesbeckia orientalis L., 2 compounds and 3 targets for Gentiana macrophylla Pall., 4 compounds and 82 targets for Salvia miltiorrhiza Bunge, 11 compounds and 108 targets for Forsythia suspensa (Thunb.) Vahl, and 11 compounds and 68 targets for Glycyrrhiza uralensis Fisch. The obtained compounds and targets were all used to construct the "drugtarget-disease" network.

\section{Network construction and pathway analysis}

The construction of target protein network of the TCM formula for a specific disease and the summary and analysis of the noteworthy features of the network would provide some important information for us to understand the drug-target interaction mechanism of the certain drugs on the specific disease. This study focused on the effect of SCG on liver fibrosis. Figure 2 shows the network for the compounds and their potential targets with color-coded nodes. The red triangles represent active chemical constituents of SCG, the blue dots represent the indirect targets for drugs, the yellow dots represent the targets of the specific disease of liver fibrosis, and the yellow squares represent the common targets of herbs and liver fibrosis. That is, the common targets are the key targets by which SCG treated liver fibrosis and are also the relatively important targets screened for further research.

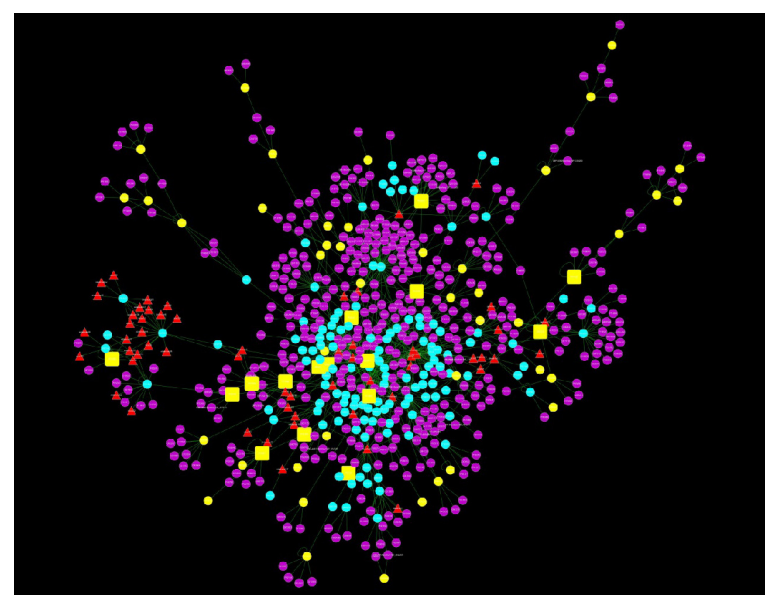

Figure 2 The main network of compound-target-disease of SCG.

Notes: The red triangles represent active chemical constituents of SCG, the blue dots represent the indirect targets for drugs, the yellow dots represent the targets of the specific disease of liver fibrosis, the yellow squares represent the common targets of herbs and liver fibrosis, and the purple dots represent the interactional proteins with the targets of liver fibrosis and drugs.

Abbreviation: SCG, San-Cao Granule. 
The purple dots represent the interactional proteins with the targets of liver fibrosis and drugs.

Table 1 indicates that 16 common targets were predicted. To further screen the more important targets, three topological parameters, "Degree", "Betweenness centrality", and "Closeness centrality", were chosen to identify candidate hepatic fibrosis targets. We identified a node as the candidate SCG target for liver fibrosis if its three topological parameters were more than twofold of the median value, respectively. After calculating the value of the three parameters for each hepatic fibrosis significant protein in the PPI network, the twofold of the median value of "Degree", "Betweenness centrality", and "Closeness centrality" was 3.02, 0.41, and 0.07, respectively. Therefore, we determined that hepatic fibrosis significant proteins with "Degree" $>3.02$, "Betweenness centrality" $>0.41$, and "Closeness centrality" $>0.07$ were candidate targets for hepatic fibrosis therapy. As a result, amyloid $\beta$ A4 protein; actin, cytoplasmic 1; transforming growth factor $\beta$-1; and fibroblast growth factor two were identified as candidate hepatic fibrosis targets.

As shown in Figure 3, 22 KEGG pathways were enriched in the ArrayTrack v.3.5.0 pathway-enrichment analysis. As we know that inflammation plays an important role in liver fibrosis, the analysis of KEGG pathways manifests that most of the pathways, such as TGF- $\beta$ signaling pathway, P53 signaling pathway, MAPK signaling pathway, Toll-like receptor signaling pathway, NOD-like receptor signaling pathway, and chemokine signaling pathway are either directly involved in immunity and inflammation or play other important roles.
Considering the "Degree", "Betweenness centrality" and "Closeness centrality" of TGF- $\beta 1$ were estimated to be relatively large, and TGF- $\beta 1$ participated in many signaling pathways that were relatively important in the obtained 22 KEGG pathways such as pathways in cancer, pancreatic cancer, chronic myeloid leukemia, cytokine-cytokine receptor interaction, cell cycle, adherens junction, and TGF- $\beta$ signaling pathway. Therefore, we selected TGF- $\beta 1$ and its downstream important proteins $\operatorname{Smad} 3$ and $\operatorname{Smad} 7$ chosen from the TGF- $\beta$ signaling pathway map in the forms of molecular networks for further verification study to validate the effectiveness of the signaling pathway for the treatment of SCG for liver fibrosis.

\section{The identification of chemical ingredients of SCG by TOF LC/MS/MS}

In our previous studies, ${ }^{17}$ we identified 15 active chemical ingredients from SCG through TOF LC/MS/MS, which were rubimaillin, purpurin, gentiopicroside, tanshinone IIA, salvianolic acid B, danshensu, glycyrrhizic acid, glycyrrhetinic acid, cryptotanshinone, alizarin, ferulic acid, formononetin, orientin, sweroside, and swertamarin as shown in Table 2.

\section{Improvement of SCG on the serum parameters of liver function and liver fibrosis in TAA-induced liver fibrotic rats}

The serum ALT, AST, ALB, and TB levels can indicate the degree of liver damage. As shown in Figure 4A, the levels of ALT, AST, and TB were significantly elevated in

Table I The targets and its relevant topological parameters and corresponding active compounds of SCG screened by network pharmacology

\begin{tabular}{|c|c|c|c|c|c|}
\hline $\begin{array}{l}\text { Direct } \\
\text { target }\end{array}$ & Target protein & Degree & $\begin{array}{l}\text { Closeness } \\
\text { centrality }\end{array}$ & $\begin{array}{l}\text { Betweeness } \\
\text { centrality }\end{array}$ & Compound \\
\hline P05067 & Amyloid $\beta$ A4 protein & 12 & 0.9167 & 0.9818 & Cryptotanshinone \\
\hline P60709 & Actin, cytoplasmic I & 9 & 0.5313 & 0.7794 & Danshensu \\
\hline P0II37 & Transforming growth factor $\beta$-I & 8 & 0.4473 & 0.4265 & Danshensu \\
\hline P09038 & Fibroblast growth factor 2 & 6 & 0.65 & 0.641 & Ursolic acid \\
\hline PII802 & Cyclin-dependent kinase 4 & II & 0.2577 & 0.0729 & Glycyrrhetinic acid \\
\hline P3 1749 & RAC- $\alpha$ serine/threonine-protein kinase & 13 & 0.2673 & 0.061 & Salvianolic acid $A$ \\
\hline P03372 & Estrogen receptor & 21 & 0.2407 & 0.0906 & Formononetin \\
\hline OI5392 & Baculoviral IAP repeat-containing protein 5 & 8 & 0.1717 & 0.0363 & Ursolic acid \\
\hline Q078।7 & Bcl-2-like protein I & 4 & 0.205 & 0.0054 & Ursolic acid \\
\hline P07550 & $\beta$-2 adrenergic receptor & 3 & $\mathrm{I}$ & 1 & II, I 2, I 3-trinorguai-6-ene- $4 \beta$, I $0 \beta$-diol \\
\hline P22303 & Acetylcholinesterase & $\mathrm{I}$ & 0.5 & 0 & Ursolic acid \\
\hline P04 I4I & Granulocyte-macrophage colony-stimulating factor & 3 & I & $\mathrm{I}$ & Glycyrrhizic acid \\
\hline POI008 & Antithrombin-III & 3 & I & 0 & Cryptotanshinone \\
\hline P62736 & Actin, aortic smooth muscle & 0 & 0 & 0 & Salvianolic acid B \\
\hline Q9UKK3 & Poly [ADP-ribose] polymerase 4 & 0 & 0 & 0 & Tanshinonell A \\
\hline P09917 & Arachidonate 5-lipoxygenase & 0 & 0 & 0 & Alizarin \\
\hline
\end{tabular}

Abbreviation: SCG, San-Cao Granule. 


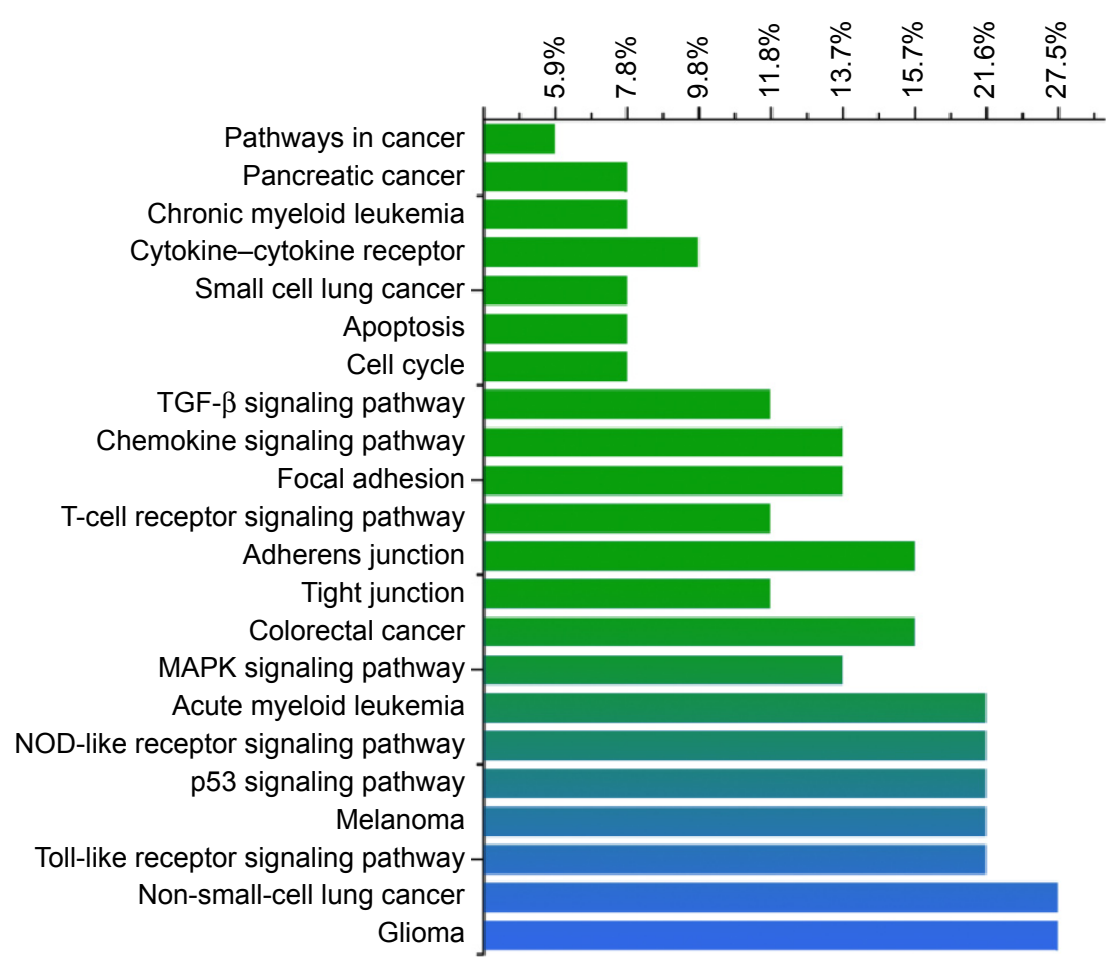

Figure 3 Potential pathways annotation of putative targets.

Notes: The bar map shows the top 22 pathways of putative targets with SCG compounds. $X$-axis represents the percentage of targets in the total pathways. Y-axis represents the names of putative pathways.

Abbreviations: TGF, transforming growth factor; MAPK, mitogen-activated protein kinase; SCG, San-Cao Granule; NOD, nucleotide-binding oligomerization domain.

TAA-treated group $(P<0.01)$, meanwhile the level of serum ALB was significantly reduced $(P<0.01)$. TAA-treated + $\mathrm{SCG}(3.6$ and $1.8 \mathrm{~g} / \mathrm{kg})$ and TAA + UDCA $(60 \mathrm{mg} / \mathrm{kg})$ markedly reduced the high serum levels of ALT, AST, and TB $(P<0.05)$, but markedly increased the level of ALB $(P<0.01)$. The improvement of SCG $(3.6$ and $1.8 \mathrm{~g} / \mathrm{kg})$ on liver injury was better than UDCA $(60 \mathrm{mg} / \mathrm{kg})$ and $\mathrm{SCG}$

Table 2 The 15 identified components in SCG

\begin{tabular}{llll}
\hline Number & $\begin{array}{l}\text { Identified } \\
\text { components }\end{array}$ & $\begin{array}{l}\text { Molecular } \\
\text { formula }\end{array}$ & Attribution \\
\hline 1 & Rubimaillin & $\mathrm{C}_{17} \mathrm{H}_{16} \mathrm{O}_{4}$ & Rubia cordifolia \\
2 & Purpurin & $\mathrm{C}_{14} \mathrm{H}_{8} \mathrm{O}_{5}$ & Rubia cordifolia \\
3 & Gentiopicroside & $\mathrm{C}_{16} \mathrm{H}_{20} \mathrm{O}_{9}$ & Gentiana macrophylla \\
4 & Tanshinone IIA & $\mathrm{C}_{19} \mathrm{H}_{18} \mathrm{O}_{3}$ & Salvia miltiorrhiza \\
5 & Salvianolic acid $\mathrm{B}$ & $\mathrm{C}_{36} \mathrm{H}_{30} \mathrm{O}_{16}$ & Salvia miltiorrhiza \\
6 & Danshensu & $\mathrm{C}_{9} \mathrm{H}_{10} \mathrm{O}_{5}$ & Salvia miltiorrhiza \\
7 & Glycyrrhizic acid & $\mathrm{C}_{42} \mathrm{H}_{62} \mathrm{O}_{16}$ & Glycyrrhiza uralensis \\
8 & Glycyrrhetinic acid & $\mathrm{C}_{30} \mathrm{H}_{46} \mathrm{O}_{4}$ & Glycyrrhiza uralensis \\
9 & Alizarin & $\mathrm{C}_{14} \mathrm{H}_{8} \mathrm{O}_{4}$ & Rubia cordifolia \\
10 & Cryptotanshinone & $\mathrm{C}_{19} \mathrm{H}_{20} \mathrm{O}_{3}$ & Salvia miltiorrhiza \\
11 & Ferulic acid & $\mathrm{C}_{10} \mathrm{H}_{10} \mathrm{O}_{4}$ & Forsythia suspense \\
12 & Formononetin & $\mathrm{C}_{16} \mathrm{H}_{12} \mathrm{O}_{4}$ & Glycyrrhiza uralensis \\
13 & Orientin & $\mathrm{C}_{21} \mathrm{H}_{20} \mathrm{O}_{11}$ & Siegesbeckia orientalis \\
14 & Sweroside & $\mathrm{C}_{16} \mathrm{H}_{22} \mathrm{O}_{9}$ & Gentiana macrophylla \\
15 & Swertamarin & $\mathrm{C}_{16} \mathrm{H}_{22} \mathrm{O}_{10}$ & Gentiana macrophylla \\
\hline
\end{tabular}

Abbreviation: SCG, San-Cao Granule.
$(0.9 \mathrm{~g} / \mathrm{kg})$. These results showed that the improvement in liver injury was better on SCG treatment (3.6 and $1.8 \mathrm{~g} / \mathrm{kg}$ ) than on UDCA treatment.

The serum levels of HA, LN, and CIV and the Hyp level in liver tissue can indicate the degree of liver fibrosis. As shown in Figures 4B and 5D, the levels of HA, LN, CIV, and Hyp were significantly increased in the TAA-treated group $(P<0.01)$. However, treatment with SCG (3.6 and $1.8 \mathrm{~g} / \mathrm{kg})$ and UDCA $(60 \mathrm{mg} / \mathrm{kg})$ markedly reduced the increased levels of HA, LN, and CIV $(P<0.05)$. The improvement with SCG treatment (3.6 and $1.8 \mathrm{~g} / \mathrm{kg}$ ) on liver fibrosis was better than that with UDCA $(60 \mathrm{mg} / \mathrm{kg})$ and SCG $(0.9 \mathrm{~g} / \mathrm{kg})$.

\section{Histological evaluation}

The histopathological changes in the liver tissues taken from all of the experiment groups were examined by HE staining. As shown in Figure 6A, the liver tissue structure of normal group exhibited regular hepatocellular arrangement with branching form and clearly showed hepatic cords. The hepatocytes had a single circular vesicular nuclei and acidophilic cytoplasm, except for a few hepatocytes that had two vesicular nuclei. The liver sections of the model group showed that many cytoplasmic vacuoles appeared in most of the 

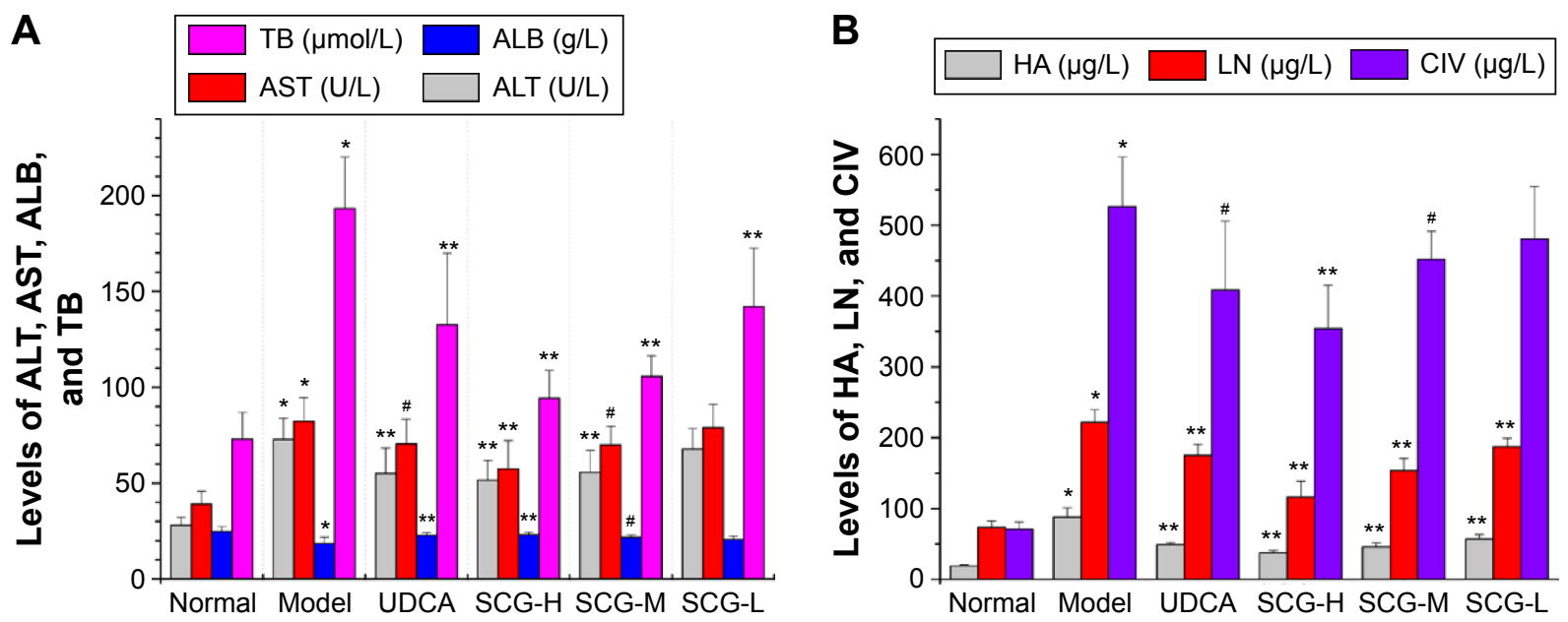

Figure 4 The serum levels of ALT, AST, ALB, TB, HA, LN, and CIV of experimental groups.

Notes: (A): ALT, AST, ALB, TB; (B): HA, LN, CIV. Data are expressed as the mean \pm SD. (SCG-H: TAA + SCG [3.6 g/kg]; SCG-M: TAA + SCG [I.8 g/kg]; SCG-L: TAA + SCG $[0.9 \mathrm{~g} / \mathrm{kg}]) . * P<0.01$ compared with normal group, $* * P<0.01$ compared with model group, ${ }^{*} P<0.05$ compared with model group.

Abbreviations: SCG, San-Cao Granule; UDCA, ursodeoxycholic acid; TAA, thioacetamide; ALT, alanine transaminase; AST, aspartate aminotransferase; ALB, albumin; TB, total bilirubin; HA, hyaluronic acid; LN, laminim; CIV, IV collagen.

hepatocytes and that portal areas were infiltrated by massive inflammatory cells, and the nuclei were stained deeply. SCG $(3.6 \mathrm{~g} / \mathrm{kg})$ administration significantly improved the disordered pathological structure of liver; SCG (1.8 and $0.9 \mathrm{~g} / \mathrm{kg}$ ) and UDCA $(60 \mathrm{mg} / \mathrm{kg})$ had moderately reduced the disorder of hepatocytes.

As shown in Figure 6B, the results of Masson's trichrome staining indicated that few collagenous fibers were observed in normal group. However, the sections of the model group had much more collagenous fibers around the central veins and portal area. SCG $(3.6 \mathrm{~g} / \mathrm{kg})$ treatment significantly reduced the content of collagenous fibers, while the treatment with SCG (1.8 and $0.9 \mathrm{~g} / \mathrm{kg})$ and UDCA $(60 \mathrm{mg} / \mathrm{kg})$ only slightly reduced the content of collagenous fibers. The results of staging scores and percentage of collagen content of liver fibrosis (Table 3 and Figure 5E) also showed that SCG $(3.6,1.8$, and $0.9 \mathrm{~g} / \mathrm{kg}$ ) and UDCA markedly alleviated the score of liver fibrosis $(P<0.01)$.

\section{Effect of SCG on $\alpha-S M A$ and CTGF}

To assess the level of $\alpha$-SMA proteins, we used immunohistochemistry and Western blot. As shown in Figure 5A and F, the immunohistochemical result showed increased expression of $\alpha$-SMA focused on fibrotic areas, central veins, and portal area; and SCG $(3.6,1.8$, and $0.9 \mathrm{~g} / \mathrm{kg})$ or UDCA significantly reduced the expression of $\alpha$-SMA. The results of Western blot indicated that SCG $(3.6,1.8$, and $0.9 \mathrm{~g} / \mathrm{kg})$ or UDCA markedly decreased the expression of $\alpha$-SMA (Figure $5 \mathrm{~B}$ ) and CTGF (Figure 5C).

\section{Effect of SCG on the expression of TGF- $\beta$ I}

TGF- $\beta 1$ is an important multifunctional cytokine because of its special role in the development of liver fibrosis. We detected the expression of TGF- $\beta 1$ using Western blot. As shown in Figure 7A and B, we found that the expression of TGF- $\beta 1$ in TAA-treated group was promoted markedly, while the administration of SCG (3.6 and $1.8 \mathrm{~g} / \mathrm{kg}$ ) treatments significantly inhibited the expression of TGF- $\beta 1$.

\section{Effect of SCG on the expression of P-Smad3, Smad3, and Smad7}

As shown in Figure 7, the expression of p-Smad3 was significantly elevated, while the expression of Smad3 was inapparent, and the expression of Smad7 was significantly reduced in TAA-treated group. SCG $(3.6$ and $1.8 \mathrm{~g} / \mathrm{kg})$ treatments remarkably attenuated the expression of $\mathrm{p}-\mathrm{Smad} 3$ and induced the expression of Smad7.

The results indicated that SCG interdicted the transmission of TGF- $\beta 1 /$ Smad through reducing the expression of TGF- $\beta 1$ and $\mathrm{p}-\mathrm{Smad} 3$ but promoting the expression of Smad7.

\section{Discussion}

To uncover the therapeutic effect on diseases and the active substances involved in the drugs, we structured an integrated model of system pharmacology to investigate the pharmacological mechanisms of SCG for treatment of liver fibrosis. In this study, we first predicted the active ingredients and 


\section{A}
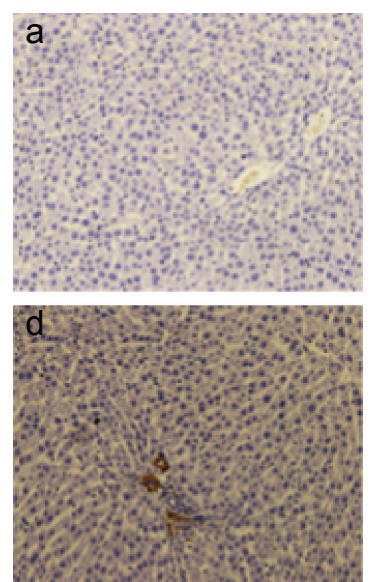

B
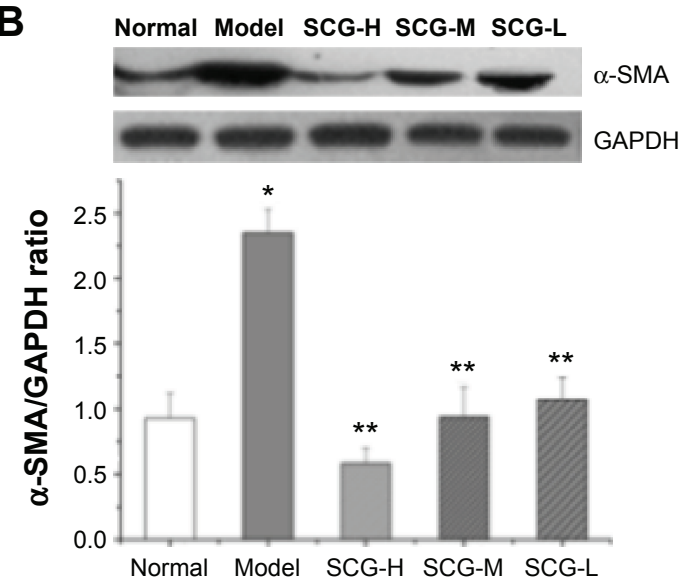

D

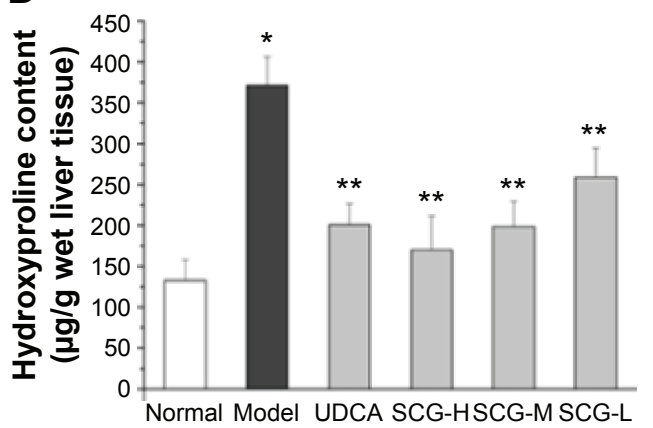

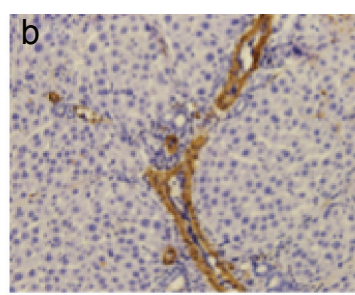
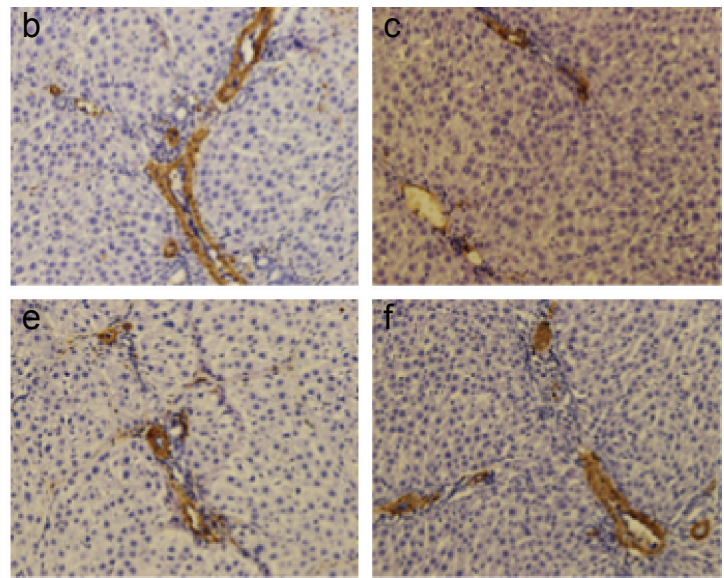

C Normal Model SCG-H SCG-M SCG-L

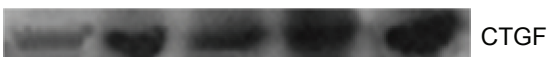

$m=0 \mathrm{GAPDH}$

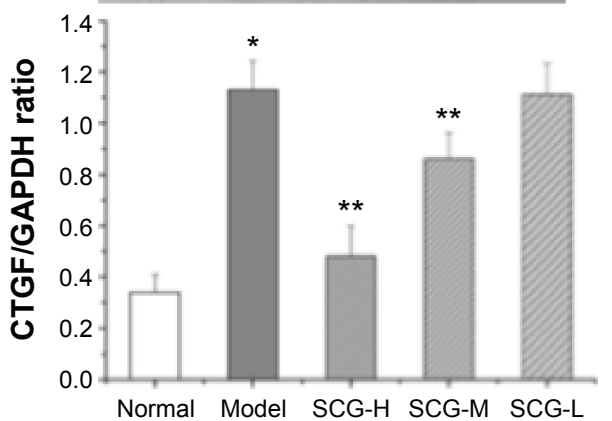

E

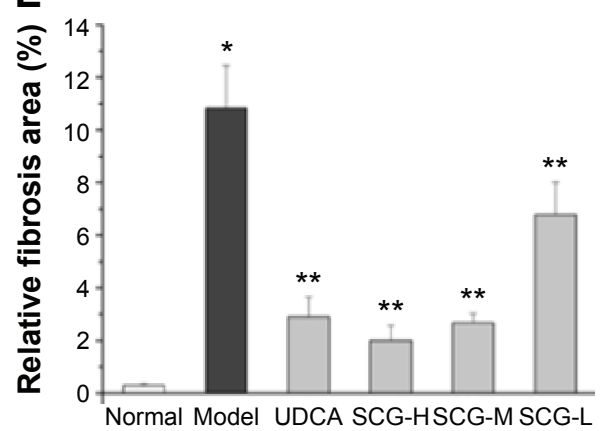

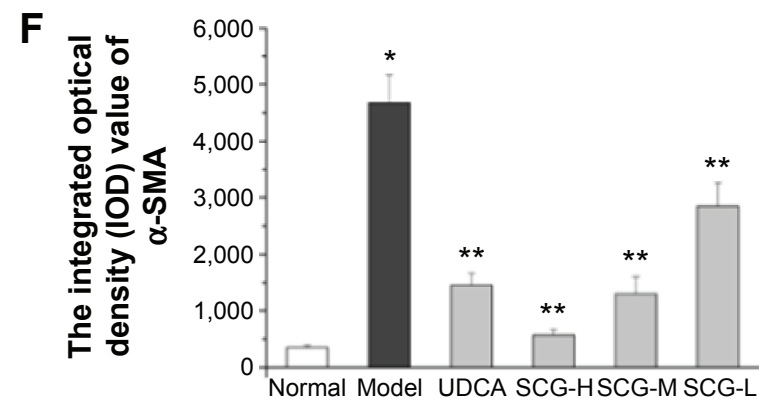

Figure $\mathbf{5}$ The effect of SCG on the expressions of $\alpha$-SMA and CTGF.

Notes: (A) The expression of $\alpha$-SMA detected by immunohistochemistry staining (a: Normal; b: Model; c: UDCA; d: SCG-H; e: SCG-M; f: SCG-L); (B, C) the expression of $\alpha$-SMA and CTGF detected by Western blot; (D) the level of Hyp in liver tissue; (E) the percentage of collagen content detected by image software through liver tissue stained by Masson (I00X); (F) the integrated optical density (IOD) value of $\alpha-S M A$. (SCG-H: TAA + SCG [3.6 g/kg]; SCG-M: TAA + SCG [1.8 g/kg]; SCG-L: TAA + SCG [0.9 $\mathrm{g} / \mathrm{kg}])$. $* p<0.0 \mathrm{I}$ compared with normal group, $* * p<0.01$ compared with model group.

Abbreviations: SCG, San-Cao Granule; UDCA, ursodeoxycholic acid; TAA, thioacetamide; SMA, smooth muscle actin; CTGF, connective tissue growth factor; GAPDH, glyceraldehyde 3-phosphate dehydrogenase; Hyp, hydroxyproline. 

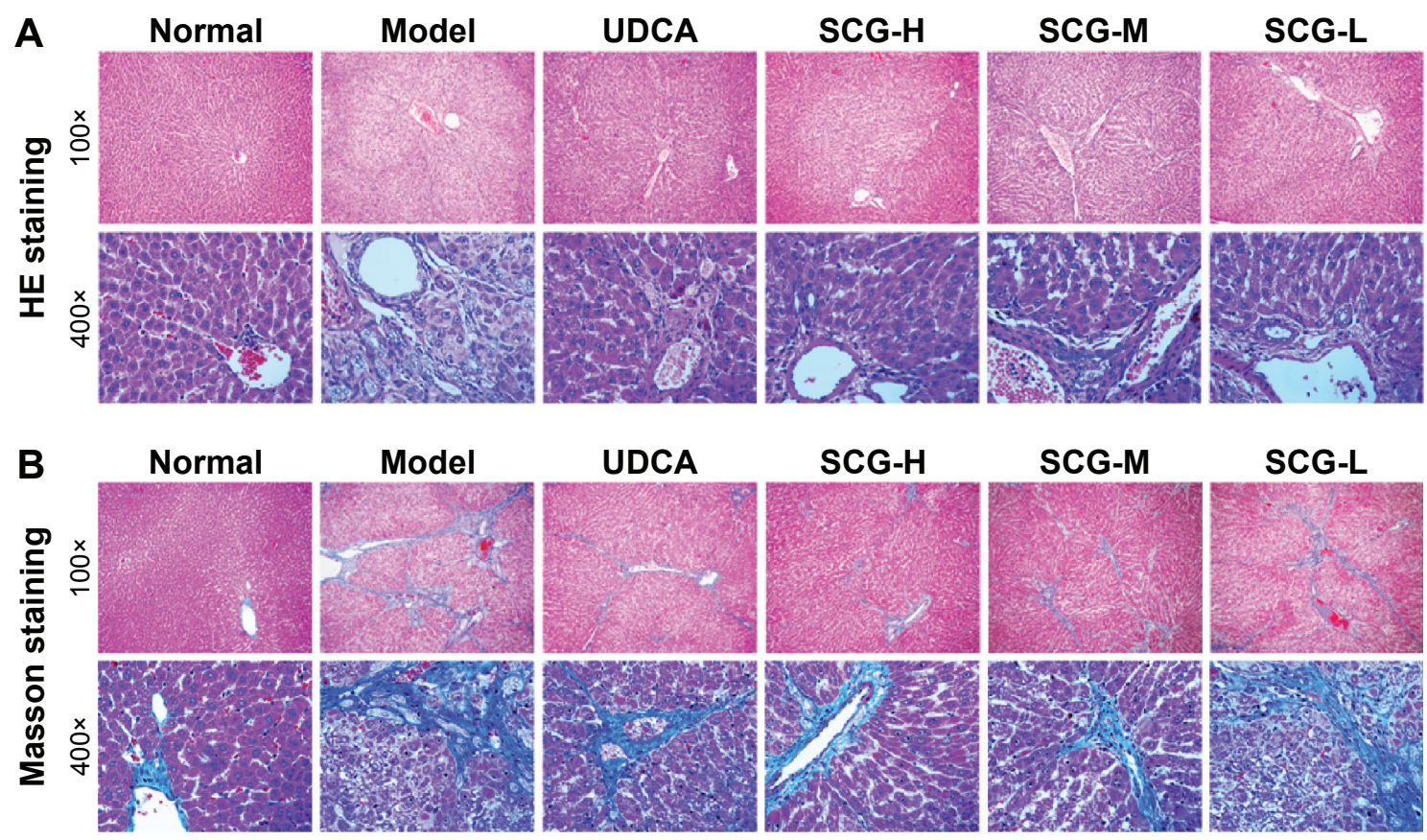

Figure 6 The effect of SCG on the pathological features of liver tissues of experimental rats stained with HE (A) and Masson (B). (SCG-H: TAA + SCG [3.6 g/kg]; SCG-M: TAA + SCG [1.8 g/kg]; SCG-L: TAA + SCG [0.9 g/ $/ \mathrm{kg}]$ )

Abbreviations: SCG, San-Cao Granule; UDCA, ursodeoxycholic acid; TAA, thioacetamide; HE, hematoxylin-eosin.

potential targets of SCG related to liver fibrosis through constructing the biological network of interaction between chemical compounds and protein targets from a molecular or systemic level.

The predicted results indicated that 11 ingredients in SCG interacted with 16 targets related to liver fibrosis in this formula. Some ingredients could modulate the same target simultaneously, which gives a good explanation of synergistic mechanisms of TCMs. It is worth mentioning that the computational models can only give hints, which still need to be verified by real experiment.

Based on the results from network pharmacology analysis, we further explored the effect of SCG on the important parameters related to liver injury and liver fibrosis by a

Table 3 The degree of hepatic fibrosis in rats

\begin{tabular}{llllllll}
\hline Groups & $\mathbf{n}$ & $\mathbf{0}$ & I & II & III & IV & $\begin{array}{l}\text { Staging scores } \\
\text { (mean } \pm \text { SD) }\end{array}$ \\
\hline Normal & 10 & 10 & 0 & 0 & 0 & 0 & 0 \\
Model & 10 & 0 & 0 & 0 & 7 & 3 & $3.3 \pm 0.48^{*}$ \\
UDCA & 10 & 0 & 1 & 4 & 5 & 4 & $2.4 \pm 0.69 * *$ \\
TAA + SCG (3.6 g/kg) & 10 & 0 & 8 & 2 & 0 & 0 & $1.2 \pm 0.42^{* *}$ \\
TAA + SCG (I.8 g/kg) & 10 & 0 & 5 & 4 & 1 & 0 & $1.6 \pm 0.68^{* *}$ \\
TAA + SCG (0.9 g/kg) & 10 & 0 & 2 & 5 & 3 & 0 & $2.1 \pm 0.73^{* *}$ \\
\hline
\end{tabular}

Notes: $* P<0.01$ compared with normal group, $* * P<0.01$ compared with model group.

Abbreviations: SCG, San-Cao Granule; UDCA, ursodeoxycholic acid; TAA, thioacetamide; SD, standard deviation.
TAA-induced liver fibrotic rat model and the key proteins analyzed by KEGG pathways and Cytoscape. The levels of ALT, AST, ALB, and TB were significantly improved by SCG, which indicated that SCG alleviated liver injury. The reduction of the levels of HA, LN, CIV, and Hyp showed that liver fibrosis induced by TAA was significantly alleviated. From the results of histological evaluation, we could find that the inflammation and damage conditions and the collagen content of hepatic tissue were markedly reduced by SCG.

It has been demonstrated that the overexpression of $\alpha$-SMA can illustrate the activation of (HCSs), which are the primary cells responsible for the development of liver fibrosis. ${ }^{18,19} \mathrm{CTGF}$ is an important fibrogenic factor synthesized by hepatocytes and HSCs and is a general mediator of the interactions between fiber-fiber, fiber-matrix, and matrix-matrix..$^{20}$ Therefore, we further evaluated the effect of SCG on the liver fibrosis by testing the expressions of $\alpha$-SMA and CTGF. Our results demonstrated that $\alpha$-SMA and CTGF were significantly reduced by SCG, which also indicated that SCG inhibited the activation of HSCs and alleviated liver fibrosis. To validate the results predicted by network pharmacology, we evaluated the expression of TGF- $\beta 1$ and its downstream signaling such as Smad3 and Smad7. The results showed that SCG significantly reduced the expression of TGF- $\beta 1$ and $\mathrm{p}-\mathrm{Smad} 3$ and increased the expression of Smad7, which indicated that SCG alleviated liver 

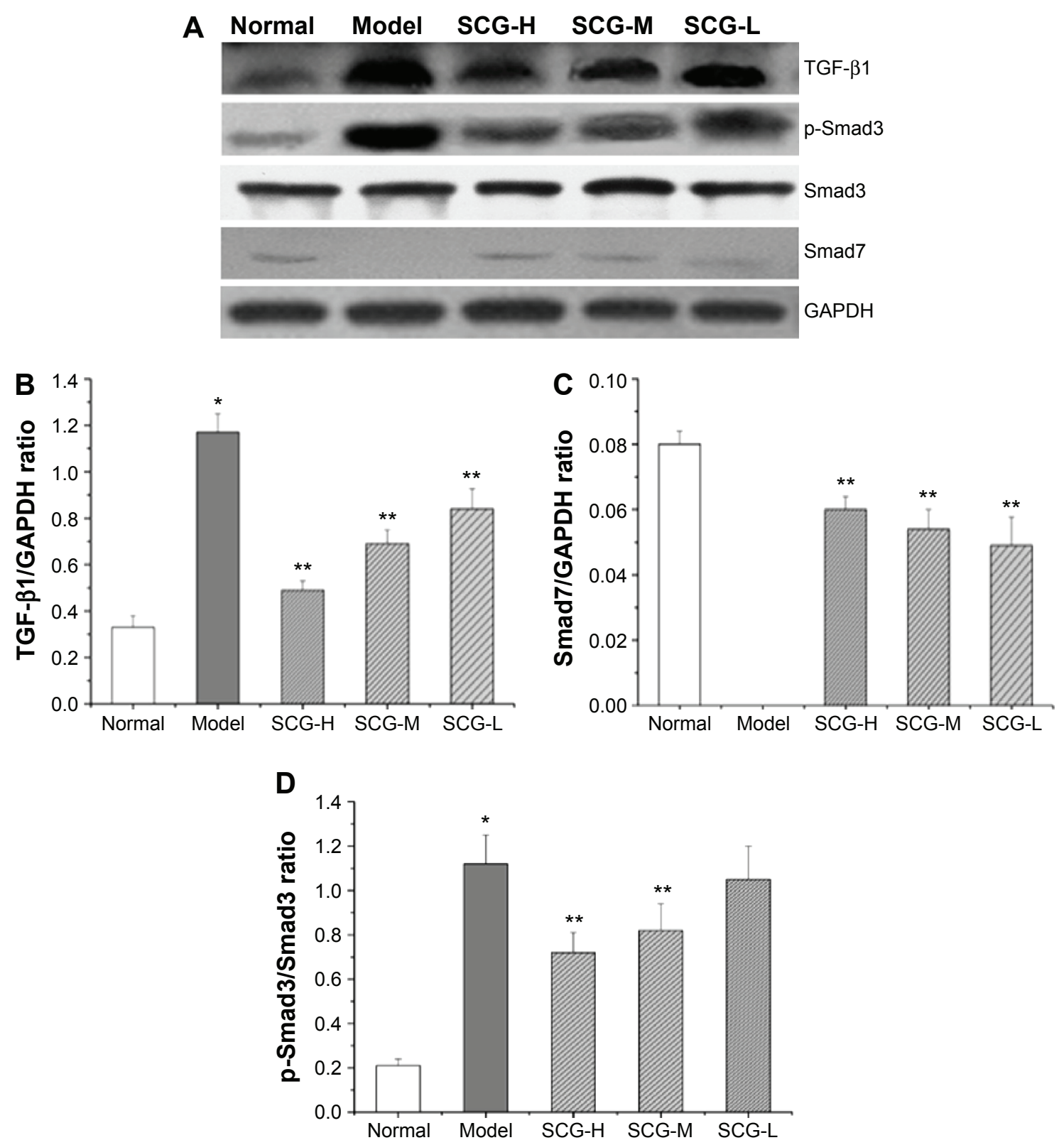

Figure 7 The effect of SCG on the expressions and semiquantitative analysis of TGF- $\beta$ I, p-Smad3, Smad3, and Smad7 detected by Western blot. Notes: (SCG-H: TAA + SCG [3.6 g/kg]; SCG-M: TAA + SCG [1.8 g/kg]; SCG-L: TAA + SCG [0.9 g/kg]). *P<0.0I compared with normal group, **P<0.0I compared with model group. (A) Western blot analysis for TGF- $\beta$ I, p-Smad3, Smad3, and Smad7; Densitometry analysis of TGF- $\beta$ I (B), Smad7 (C), and p-Smad3/Smad3 (D) on Western blot. Abbreviations: SCG, San-Cao Granule; TAA, thioacetamide; TGF- $\beta$ I, transforming growth factor $\beta$ I; GAPDH, glyceraldehyde 3-phosphate dehydrogenase.

fibrosis through the action mechanisms predicted by network pharmacology analysis. Meanwhile, tanshinone IIA, ${ }^{21}$ danshensu, ${ }^{22}$ salvianolic acid $\mathrm{B},{ }^{23}$ glycyrrhetinic acid, ${ }^{24}$ and glycyrrhizic acid ${ }^{20}$ have antifibrosis effects, as reported by some previous studies. The five aforementioned components were also predicted as the active components of SCG, treating liver fibrosis by our method of network pharmacology. Therefore, network pharmacology can be considered to be an effective way to predict and discover new medicines treating specific diseases.
The understanding of the mechanism of Chinese herbal formulas is necessary for its modernization, and the integrative and holistic ideology of network pharmacology is consistent with the essence of Chinese herbal formulas in treatments. Therefore, network pharmacology can be applied to the action mechanism research of Chinese herbal formulas and promote its modernization. Moreover, the ability of revealing correlations between drugs and diseases can help network pharmacology develop into a new strategy for new drug research and development. 


\section{Acknowledgments}

This work was supported by the Major Projects of the National Science and Technology (no 2012ZX10005010002-002) and the National Natural Science Foundation Project of China (no 81303120) and (no 81173571).

\section{Disclosure}

The authors report no conflicts of interest in this work.

\section{References}

1. Ling H, Roux E, Hempel D, et al. Transforming growth factor $\beta$ neutralization ameliorates pre-existing hepatic fibrosis and reduces cholangiocarcinoma in thioacetamide-treated rats. PLoS One. 2013;8:e54499.

2. Ahmed SK, Mohammed SA, Khalaf G, Fikry H. Role of bone marrow mesenchymal stem cells in the treatment of CCL4 induced liver fibrosis in albino rats: a histological and immunohistochemical study. Int J Stem Cells. 2014;7:87-97.

3. Shaker ME, Salem HA, Shiha GE, Ibrahim TM. Nilotinib counteracts thioacetamide-induced hepatic oxidative stress and attenuates liver fibrosis progression. Fundam Clin Pharmacol. 2011;25:248-257.

4. Tao YY, Yan XC, Zhou T, Shen L, Liu ZL, Liu CH. Fuzheng Huayu recipe alleviates hepatic fibrosis via inhibiting TNF- $\alpha$ induced hepatocyte apoptosis. BMC Complement Altern Med. 2014;14:449-462.

5. Zhao XK, Cheng ML, Wu RM, et al. Effect of Danshao Huaxian capsule on Gremlin and bone morphogenetic protein-7 expression in hepatic fibrosis in rats. World J Gastroenterol. 2014;20:14875-14883.

6. Chien SC, Chang WC, Lin PH, et al. A Chinese herbal medicine, Jia-WeiXiao-Yao-San, prevents dimethylnitrosamine-induced hepatic fibrosis in rats. ScientificWorldJournal. 2014;2014:217525.

7. Yao XX, Jiang SL, Tang YW, Yao D, Yao X. Efficacy of Chinese medicine Yi-gan-kanggranule in prophylaxis and treatment of liver fibrosis in rats. World J Gastroenterol. 2005;11:2583-2590.

8. Sheng SJ, Wang JX, Wang LR, et al. Network pharmacology analyses of the antithrombotic pharmacological mechanism of Fufang Xueshuantong Capsule with experimental support using disseminated intravascular coagulation rats. J Ethnopharmacol. 2014;154:735-744.

9. Li XX, Xu X, Wang JN, et al. A system-level investigation into the mechanisms of Chinese traditional medicine: compound Danshen formula for cardiovascular disease treatment. PLoS One. 2012;7: e43918.
10. Chen, CTC. TCM Database@Taiwan: the world's largest traditional Chinese medicine database for drug screening in silico. PLoS ONE. 2011;6(1):e15939.

11. Ye H, Ye L, Kang H, et al. HIT: linking herbal active ingredients to targets. Nucleic Acids Res. 2011;39:D1055-D1059.

12. Chen X, Ji ZL, Chen YZ. TTD: Therapeutic Target Database. Nucleic Acids Res. 2002;30(1):412-415.

13. Amberger JS, Bocchini CA, Schiettecatte F, et al. OMIM.org: Online Mendelian Inheritance in Man $\left(\mathrm{OMIM}^{\circledR}\right)$, an online catalog of human genes and genetic disorders. Nucleic Acids Res. 2015;43: D789-D798.

14. Yang M, Chen JL, Xu LW, Ji G. Navigating traditional Chinese medicine network pharmacology and computational tools. Evid Based Complement Alternat Med. 2013;2013:731969.

15. Li S, Zhang ZQ, Wu LJ, Zhang XG, Li YD, Wang YY. Understanding ZHENG in traditional Chinese medicine in the context of neuro-endocrine-immune network. IET Syst Biol. 2007;1:51-60.

16. He JY, Ge WH, Chen Y. Iron deposition and fat accumulation in dimethylnitrosamine-induced liver fibrosis in rat. World J Gastroenterol. 2007;13:2061-2065.

17. Wei SZ, Luo SQ, Wang J, et al. San-Cao Granule ameliorates hepatic fibrosis through high mobility group box-1 protein/smad signaling pathway. Chin J Integr Med. 2015. Epub ahead of print.

18. Matsue Y, Tsutsumi M, Hayashi N, et al. Serum osteopontin predicts degree of hepatic fibrosis and serves as a biomarker in patients with hepatitis C virus infection. PLoS One. 2015;10:e118744.

19. Lin HJ, Chen JY, Lin CF, et al. Hepatoprotective effects of Yi Guan Jian, an herbal medicine in rats with dimethylnitrosamine-induced liver fibrosis. J Ethnopharmacol. 2011;134:953-960.

20. Liang B, Guo XL, Jin J, Ma YC, Feng ZQ. Glycyrrhizic acid inhibits apoptosis and fibrosis in carbon-tetrachloride-induced rat liver injury. World J Gastroenterol. 2015;21:5271-5280.

21. Meng Z, Meng L, Wang K, et al. Enhanced hepatic targeting, biodistribution and antifibrotic efficacy of tanshinone IIA loaded globin nanoparticles. Eur J Pharm Sci. 2015;73:35-43.

22. Qu W, Huang H, Li K, Qin C. Danshensu-mediated protective effect against hepatic fibrosis induced by carbon tetrachloride in rats. Pathol Biol (Paris). 2014;62:348-353.

23. Li S, Wang L, Yan X, et al. Salvianolic acid B attenuates rat hepatic fibrosis via downregulating angiotensin II signaling. Evid Based Complement Alternat Med. 2012;2012:160726.

24. Chen S, Zou L, Li L, Wu T. The protective effect of glycyrrhetinic acid on carbon tetrachloride-induced chronic liver fibrosis in mice via upregulation of Nrf2. PLoS One. 2013;8:e53662.

\section{Publish your work in this journal}

Drug Design, Development and Therapy is an international, peerreviewed open-access journal that spans the spectrum of drug design and development through to clinical applications. Clinical outcomes, patient safety, and programs for the development and effective, safe, and sustained use of medicines are a feature of the journal, which

\section{Dovepress}

has also been accepted for indexing on PubMed Central. The manuscript management system is completely online and includes a very quick and fair peer-review system, which is all easy to use. Visit http://www.dovepress.com/testimonials.php to read real quotes from published authors. 